\title{
Low-cost equipment for schedule generation I: Fixed- and random-ratio schedules
}

\author{
L. J. AYLESBURY and R. GLYNN OWENS \\ University of Oxford, Oxford OX\$ $7 \boldsymbol{J X}$, England
}

\begin{abstract}
The use of complementary metal oxide semiconductor (CMOS) technology to produce fixed-ratio and random-ratio schedules is described. Such a technology implies several advantages over traditional methods of construction, especially with respect to cost and complexity. Suitable interfaces for linking with everyday laboratory equipment are also described.
\end{abstract}

The recent rapid development of complementary metal oxide semiconductor (CMOS) integrated circuitry provides the behavioral researcher with the ability to produce low-cost equipment for the generation of reinforcement schedules. Using a modular approach, all fundamental schedules of reinforcement can be individually produced and subsequently combined through standard logic functions to provide more complex schedules. Moreover, the peculiar characteristics of CMOS permit the production of such equipment at very little cost, and with remarkable simplicity and reliability.

The equipment described here produces three independent variable-interval schedules, three randomratio schedules, five fixed-ratio schedules, three fixedinterval schedules, three differential-reinforcement-oflow-rate (drl) schedules, and a variable-ratio schedule. Any or all of these may be interconnected via a network of patch leads through a panel supplying flip-flops, AND gates, OR gates, and inverters, to provide a complete range of behavioral schedules. The total cost of the equipment, including hardware (plugs and sockets, etc.), is less than $£ 350$ (approximately $\$ 600$ ). The selection of modules can be made according to the specific requirements of the user, enabling simple or more complex equipment to be produced at a cost to suit the user. Time taken to build the equipment depends on the degree of complexity required, but a single module can usually be built in less than a day.

CMOS technology is simple to work with (the equipment can be assembled in any reasonable workshop) and to operate. Modules act, in most cases, with a single output and input, together with the appropriate knob(s) or switch(es) to set the parameters of the schedule. Thus, the simple schedule can be set up in minutes by students unfamiliar with any laboratory equipment. Other advantages include low power

This work was completed while the second author was in receipt of a grant from the Medical Research Council of Great Britain, to whom thanks are due. Reprints may be obtained from Glynn Owens, University of Oxford, Department of Psychiatry, Warneford Hospital, Oxford, OX3 7JX, England. consumption (the logic can, in principle, be battery operated), high noise immunity (especially when compared to other logic families), and flexibility with regard to supply voltages $[3-15 \mathrm{~V}$ as compared with 4.75-5.25 V for transistor-transistor logic (TTL)] . CMOS is tolerant of wide temperature variations.

The use of CMOS provides a cheap extension to the capabilities of computerized systems when the latter are working at full capacity, and any damage can be repaired by a technician rather than a specialist computer engineer. For students, the difficulties of programming are obviated, since the system is largely self-explanatory.

Disadvantages of working with CMOS are few. Perhaps the greatest disadvantage is sensitivity to static electricity; CMOS integrated circuits are normally supplied embedded in conductive foam, in which they should remain until ready for use. Speed of operation is slow compared to TTL, but this is unlikely to be a problem in the applications of the type cited here (typical rise time is quoted as $25 \mathrm{nsec}$ ). Finally, it is desirable to observe a few operating precautions: Unused inputs should be connected to a supply line rather than left floating, and signals should not be present at the inputs when the equipment is switched off. Such precautions are only relevant to the setting-up procedure. Once an experiment is running, no difficulty should be encountered.

The present paper describes the production of equipment for generating fixed- and random-ratio schedules, together with the associated power supplies and interfacing. Subsequent papers will deal with the production of drl, fixed- and variable-interval, and variable-ratio schedules, together with associated logic connections.

\section{THE FIXED-RATIO (FR) SCHEDULE}

Figure 1 shows the logical structure of the FR circuit, producing a single output pulse after some fixed number of input pulses, here capable of providing ratios from $1: 1$ to $1: 999 . Q_{1}-Q_{3}$ are divide-by-10 counters, their outputs being connected via 10-way thumbwheel 


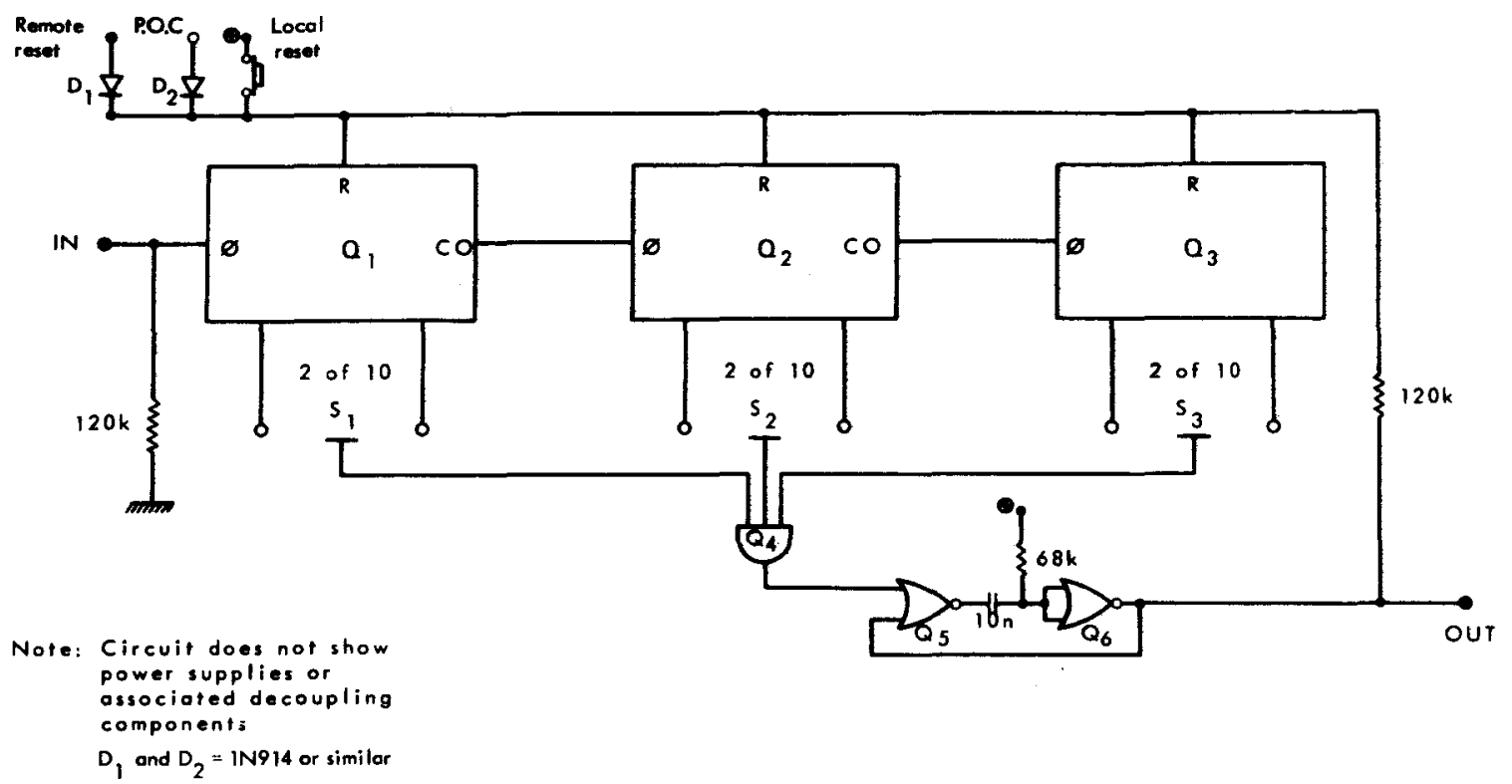

Figure 1. Logical structure of the fixed-ratio circuit.

switches to the three-input AND gate, $Q_{4}$. Thus, when each counter receives the criterion number of pulses set by the switch, all inputs to the AND gate are high, providing an input to the monostable formed by $Q_{5}$, and $\mathrm{Q}_{6}$ and associated components, and a $1 \mathrm{-msec}$ pulse at the output. This pulse also resets the three counters via the 120-k resistor. Resetting is also possible via the Power On Clear (POC), which resets all counters when the equipment is first switched on, by means of the push-button local reset, or by means of the remote reset input.

Figure 2 shows the circuit diagram to build the module. The counters are provided by the three $4017 \mathrm{~s}$, the three-input AND by the 4073, and the inverting OR gates, $Q_{5}$ and $Q_{6}$, by the 4001 .

\section{THE RANDOM-RATIO (RR) SCHEDULE}

Figure 3 gives the logical structure of the RR circuit, in which any given input pulse has a constant probability $p$ of providing an output pulse. The module is controlled by the two-input AND gate, $Q_{7}$. One input is connected directly to the incoming pulses, and the other input determines whether the pulses are transmitted to the output. Control is provided by a variable-duty cycle oscillator, formed by Inverters $Q_{1}-Q_{6} \cdot Q_{1}$ and $Q_{2}$ are connected as an astable multivibrator with a period of $50 \mathrm{msec}$, and are connected to a retriggerable monostable formed by Inverters $Q_{3}$ and $Q_{4}$. Each time the input to $Q_{3}$ goes low, $D_{1}$ conducts and charges the 47-n capacitor to the supply voltage. $Q_{4}$ 's output will then be low and will stay so until the 47-n capacitor discharges through the ratio potentiometer, causing $\mathrm{Q}_{4}$ 's output to go high. $Q_{5}$ and $Q_{6}$ shape the output giving fast edges. Thus, the ratio potentiometer varies the length of the output pulse from $0 \%$ to $100 \%$ of the duty cycle, the latter being obtained when discharge time is longer than retriggering time. The probability of an output pulse is determined by the setting of the potentiometer: The short cycle time of the multivibrator is such that a responding organism is unlikely to obtain the degree of regularity in responding necessary to "lock on" to the cycle. Figure 4 gives the wiring diagram for the circuit.

\section{POWER SUPPLIES AND PULSE FORMING}

The use of CMOS does not involve complex or especially stable power supplies. The power supply illustrated in Figure 5 has proved convenient with the prototype equipment. It provides two similar outputs: One may be used to drive the logic, and the other is available for the operation of other equipment interfaced with the logic. The transformer used is largely determined by the equipment to be operated in conjunction with the logic. For loads greater than $100 \mathrm{~mA}$, the regulator $R_{2}$ should be replaced by one with a higher current rating.

Suitable pulses for operating the circuits may be obtained from the circuit shown in Figure 6. Switch 1 is a standard changeover switch operated by the response key or lever. The circuit formed by $Q_{1}$ and $\mathrm{Q}_{2}$ eliminates the effects of contact bounce in the switch and, on operation of the switch, feeds pulses to the monostable formed by Gates $Q_{3}$ and $Q_{4}$. This monostable, besides providing the output pulse $(1 \mathrm{msec})$, also eliminates holding of the switch in one position, since the circuit requires full cycling of the input for firing. Figure 7 illustrates the use of the CD4001 integrated circuit to provide such a circuit using few external components. 


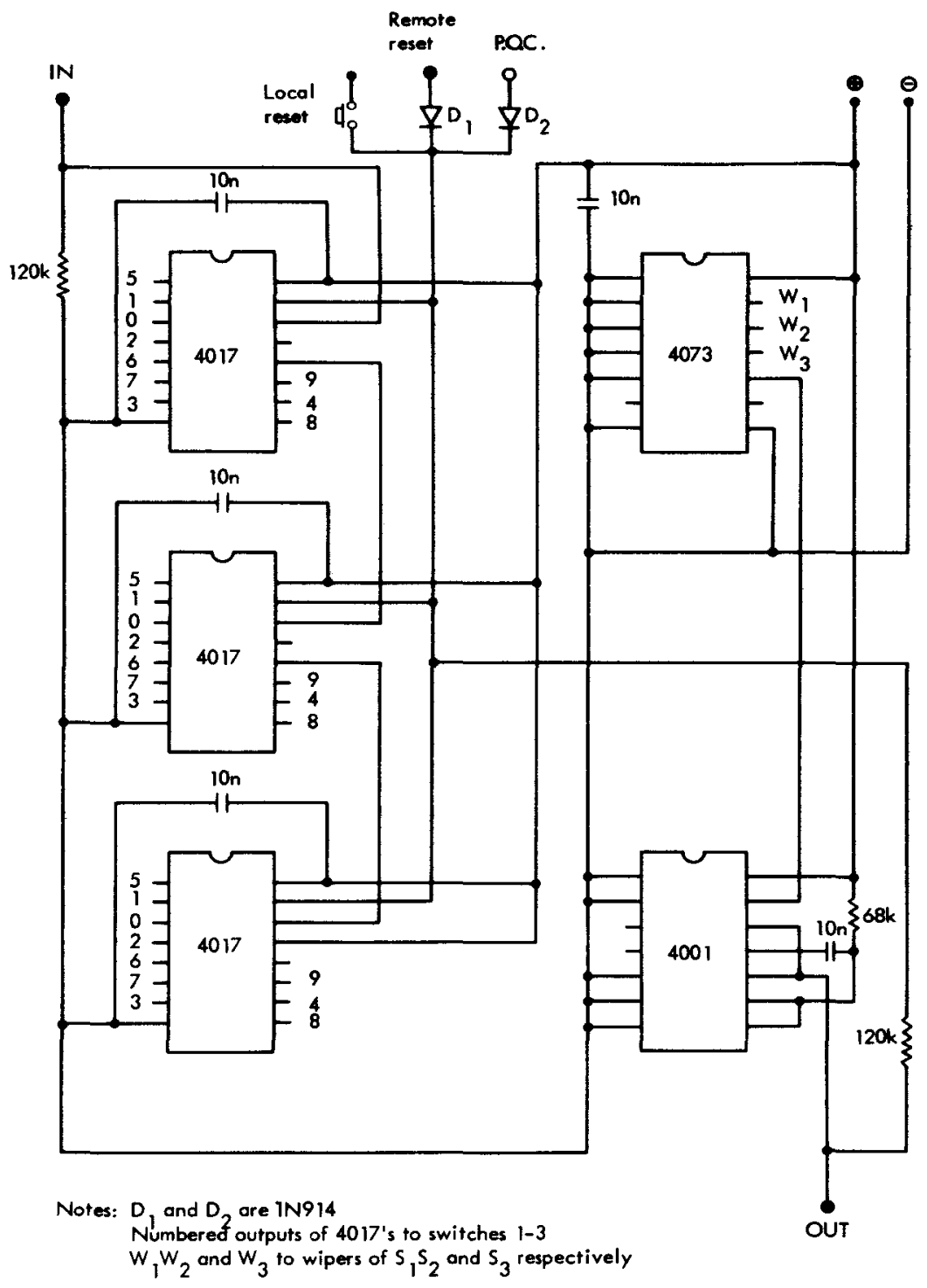

Figure 2. Circuit diagram of fixed-ratio counter.

The output from the logic may be used to drive practically any kind of equipment the experimenter may wish to use. In general, such equipment requires a pulse, or the closure of a switch. Figure 8 shows a pulse-forming circuit that provides a positive-going pulse, as required for the operation of some cumulative recorders. The duration of the output pulse is equal to the duration of the input pulse, and goes to $+12 \mathrm{~V}$ from $-10 \mathrm{~V}$. Other pulses may be produced using similar circuits.

For most applications, a simple switch closure will suffice, and this can easily be provided with a relay, as shown in Figure 9. The figure shows the use of an integrated circuit timer to operate a $24-\mathrm{V}$ relay, keeping the relay closed for times of from less than $1 \mathrm{sec}$ to several minutes, depending on the values of the components used. Although a relay is shown, any type of load may be driven using the appropriate interconnections.

The major act of producing CMOS equipment lies in the hardware associated with the logic, rather than in the electronic components themselves. Over $80 \%$ of the cost of the prototype equipment was for hardware, plugs, sockets, and the like. Despite low cost, the equipment has worked reliably in a wide range of experiments. 


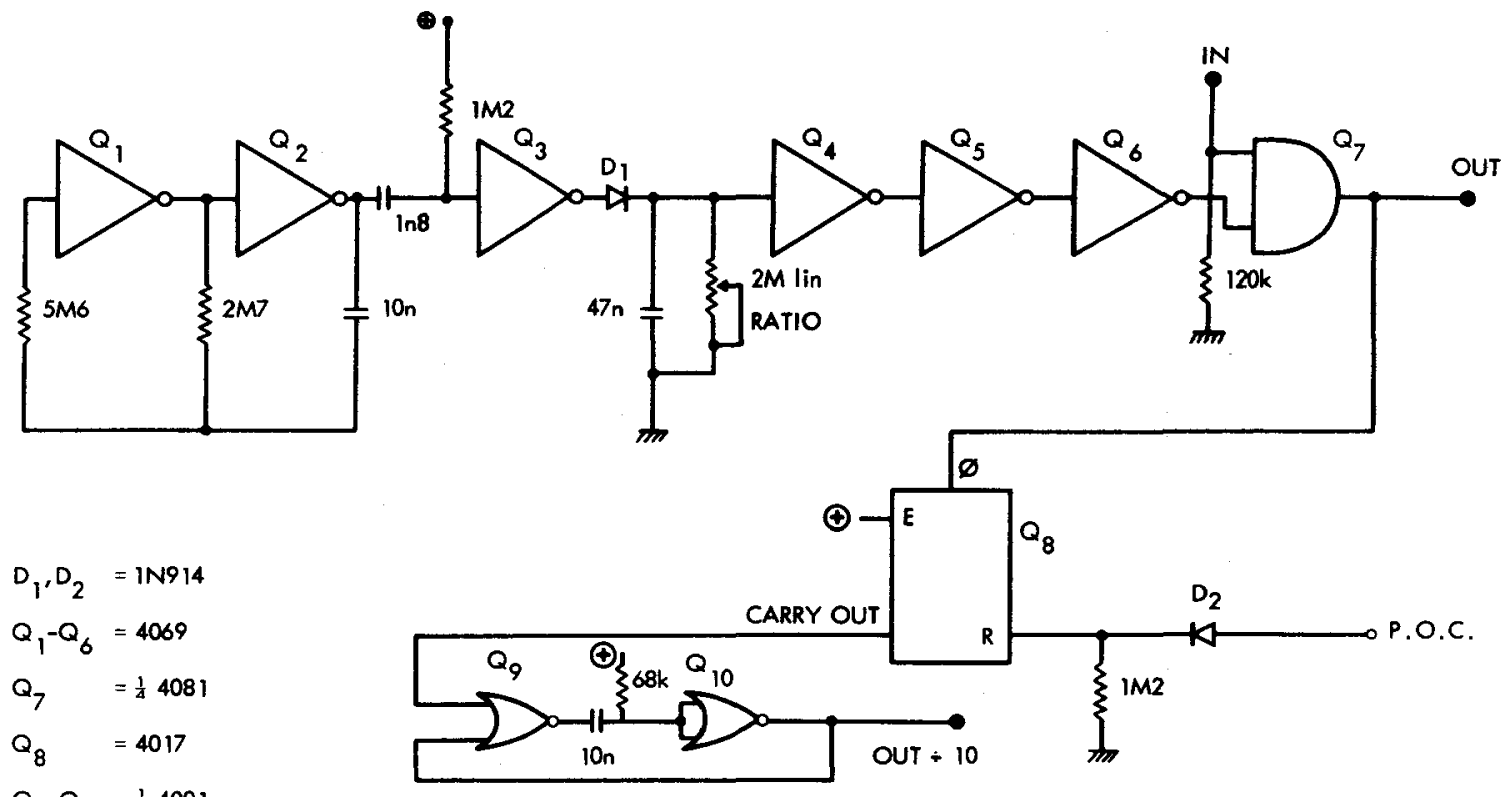

$Q_{9}, Q_{10}=\frac{1}{2} 4001$

Cir euit does not show power supply connection

or associoted decoupling components

Figure 3. Logical structure of random-ratio circuit.

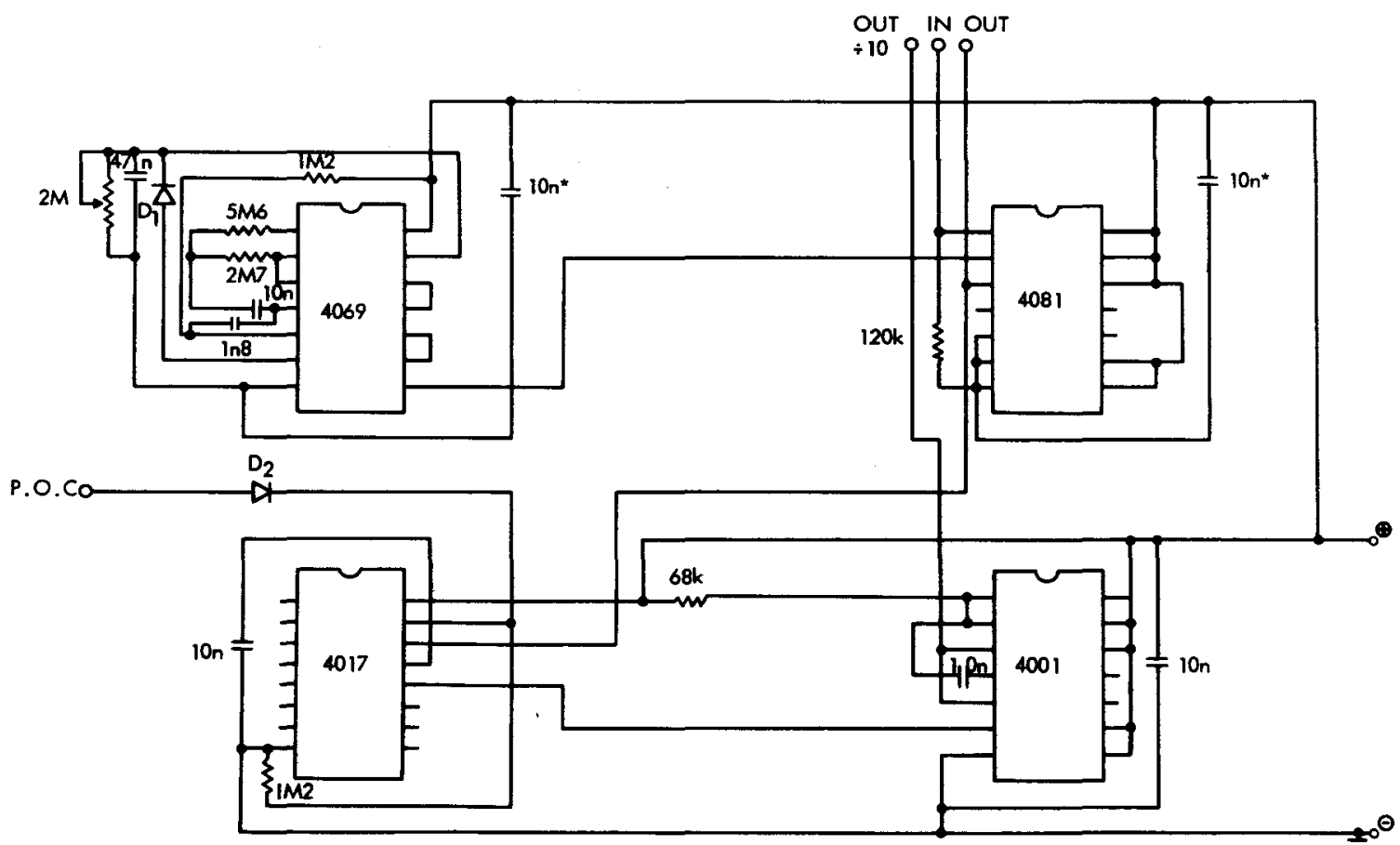

$D_{1}$ and $D_{2}=1 N 914$ or similar

*Indicates decoupling capacitors

Figure 4. Circuit diagram for random-ratio generator. 


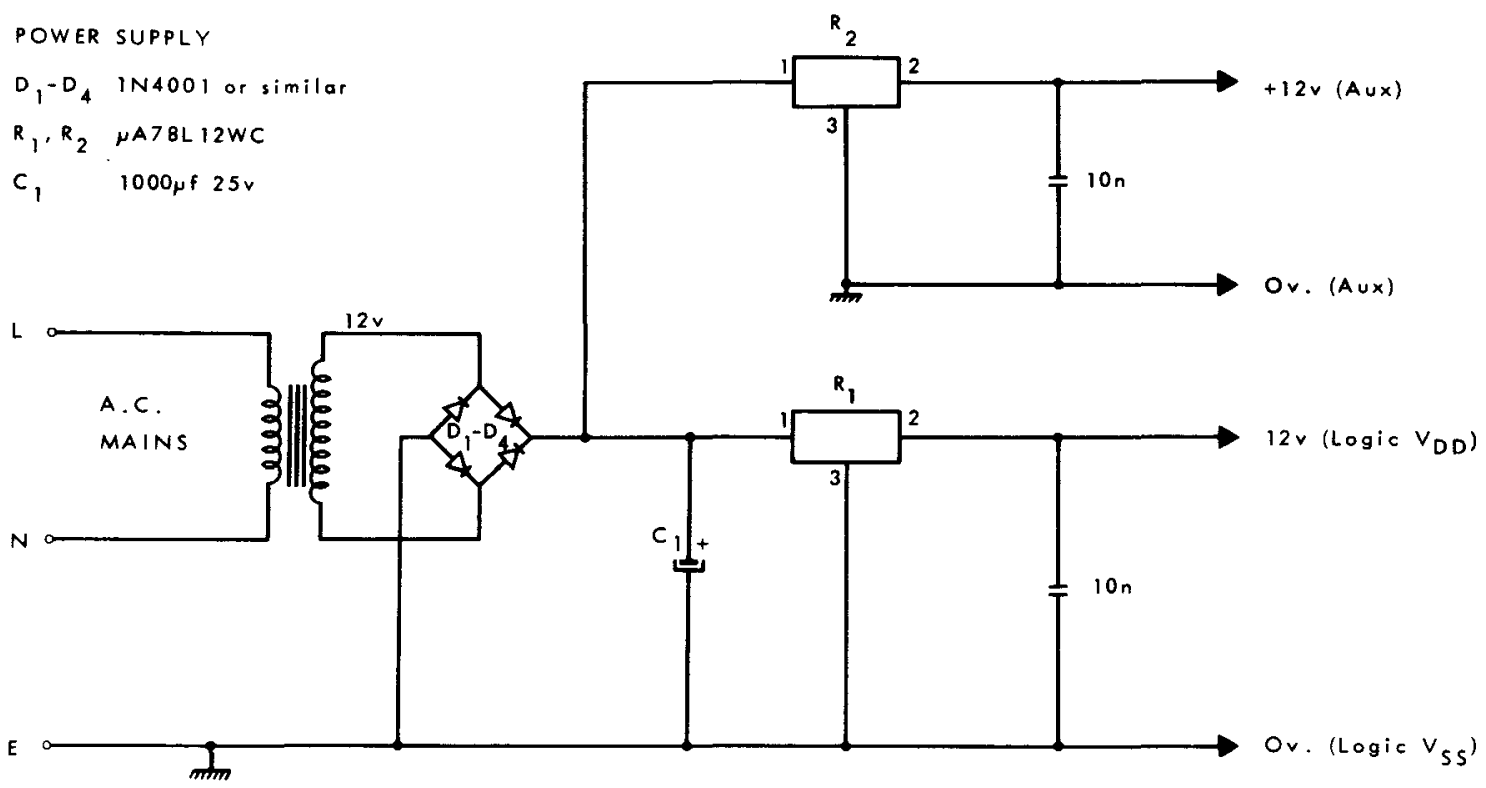

Figure 5. Power supply circuit.

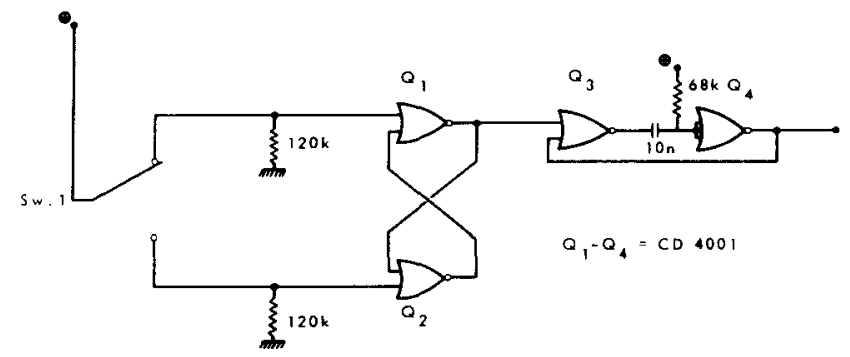

Figure 6. Pulse-forming circuit for response keys.

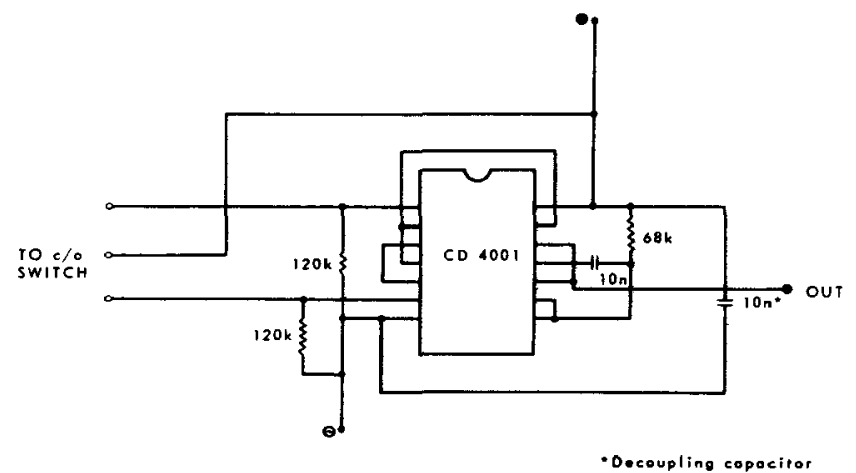

Figure 7. Integrated circuit pulse former for response keys.

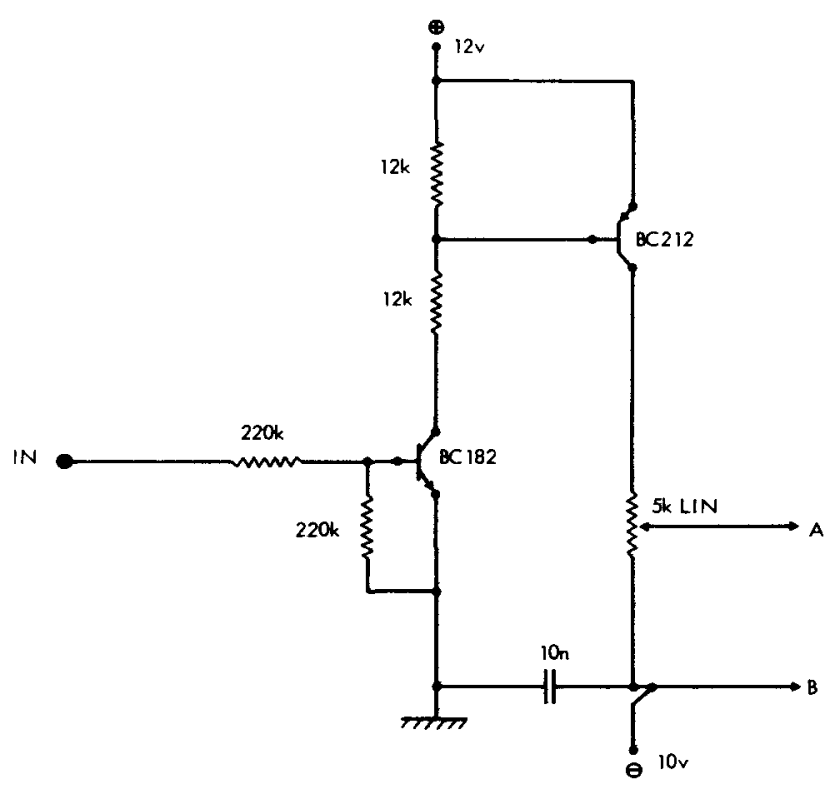

Figure 8. Interface for driving Gerbrands G-3110 cumulative recorder. 


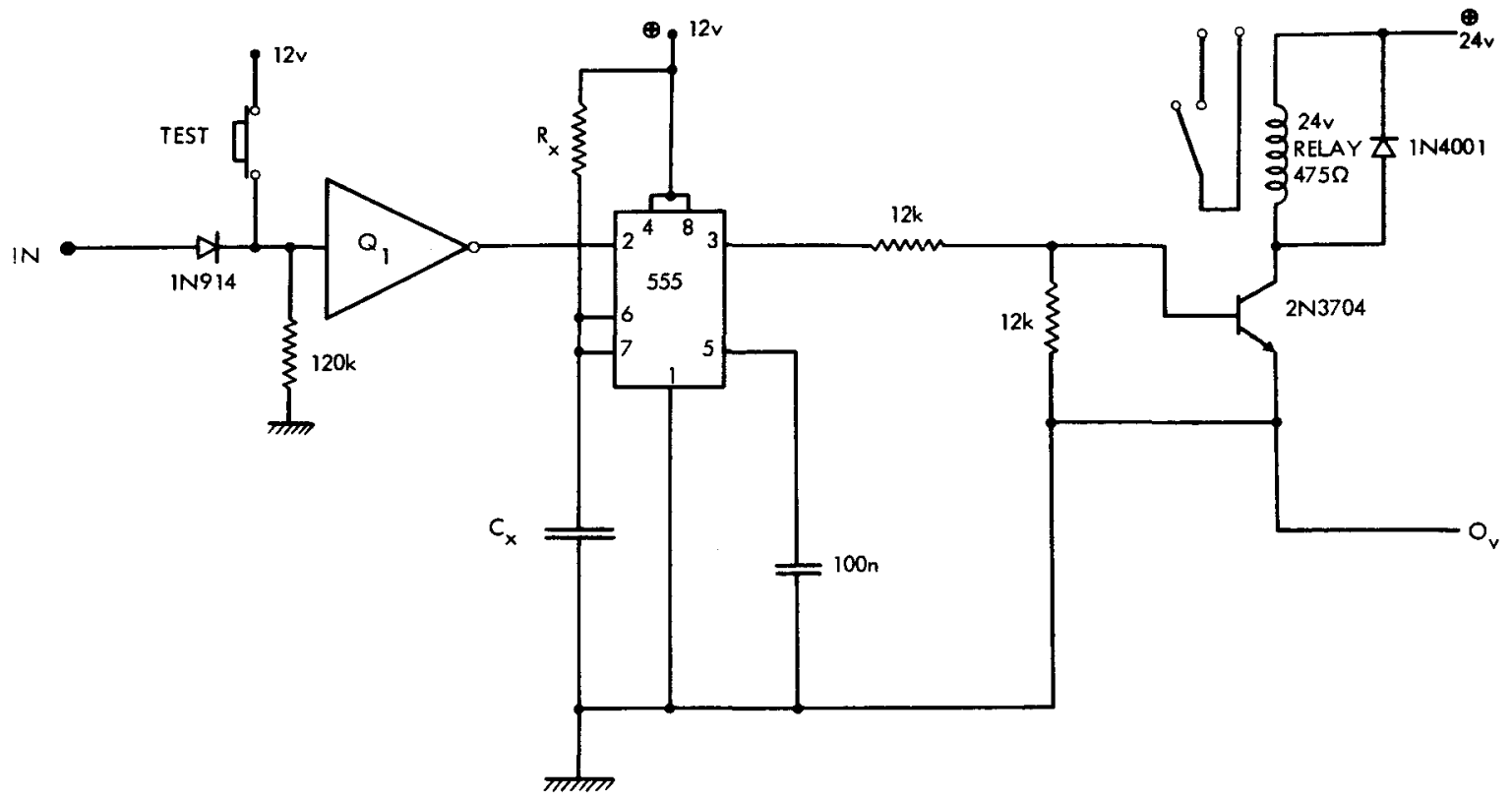

Figure 9. Interface between logic and external equipment. $Q_{1}=1 / 64069$. The time for which the relay is closed is given by $t=1.1 C R$, where $t=$ time in seconds, $R$ is the value of $R x$ in ohms, and $C$ is the value of $C x$ in farads.

(Received for publication October 18, 1976; revision accepted December $3,1976$. ) 